\title{
A Systemic Review: Structural Mechanism of SARS-CoV- 2A and Promising Preventive Cure by Phytochemicals
}

\author{
Dr. Roli Jain", Dr. Sandeep Shukla, Dr. Neeti Nema and Dr. Archna Panday \\ Department of Chemistry, Dr. Hari Singh Gour Central University, India \\ *Corresponding author: Roli Jain, Department of Chemistry, Dr. Hari Singh Gour Central University, India
}

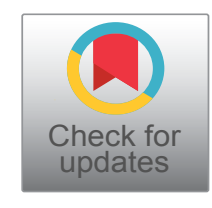

\begin{abstract}
The novel coronavirus 2019 has recently emerged as a human pathogen in the city of Wuhan in China's Hubei province, causing fever, severe respiratory illness, and pneumonia disease recently named COVID-19 [1,2].

The world experienced the outbreaks of coronavirus infection that threaten global pandemic in 2002-2003 by Severe Acute Respiratory Syndrome (SARS) and in 2011 by Middle East Respiratory Syndrome (MERS). The genomic sequence of SARS-CoV-2 showed similar, but distinct genome composition of SARS-CoV and MERS-CoV.

Phytochemicals are a powerful group of compounds, belonging to secondary metabolites of plants and including a diverse range of chemical entities such as polyphenols, flavonoids, steroidal saponins, organ Sulphur compounds, and vitamins. The potential biological benefits such as antioxidant, anti-inflammatory, anticancer, antibacterial, antifungal and antiviral activities.

The ideal technology would be vaccine that gives lifelong immunity with a single dose. When whole world community of scientist working hard to find out clinical solution of this problem so mean time we have to work with what we have in hand the best prevention approach and the phytonutrient could be a one of them.
\end{abstract}

\section{Keywords}

COVID-19, Phytochemical, Antiviral, Curcumin, Lock \& Key mechanism

\section{Introduction}

The novel coronavirus 2019 has recently emerged as a human pathogen in the city of Wuhan in China's Hubei province, causing fever, severe respiratory illness, and pneumonia disease recently named COVID-19 [1,2].

The world experienced the outbreaks of coronavirus infection that threaten global pandemic in 2002-2003 by Severe Acute Respiratory Syndrome (SARS) and in 2011 by Middle East Respiratory Syndrome (MERS). The genomic sequence of SARS-CoV-2 showed similar, but distinct genome composition of SARS-CoV and MERSCoV $[3,4]$.

The World Health Organization (WHO) also declared a global emergency on January $31^{\text {st }}$ due to increasing concerns over its fast spread, and on March $11^{\text {th }}$ the disease was recognized as a pandemic.

As of today, 26 Apr 2020 around 3 million cases were reported that infected by coronavirus with 200,000 deaths while 840,000 has been recovered. The distribution of total cases and daily projection of cases given in [5] (Figure 1).

On an average corona hit the $1.5 \%$ to 2020 global GDP and $0.2 \%$ to long-run global GDP. We forecast a muted long-term impact because damage to productive capacity will be small, plus economic confidence should quickly return once the virus subsides [6,7] (Figure 2).

Human pathogenic subtypes of $\mathrm{CoV}$ are associated with mild clinical symptoms. However, severe acute respiratory syndrome related coronavirus (SARS-CoV) and Middle East respiratory syndrome coronavirus (MERSCoV) are the two notable exceptions. In 2012, MERS-CoV was first detected in Saudi Arabia. It was responsible for 2,494 confirmed cases, which led to 858 fatalities [8]. In 2002 , a subtype of the beta-COV rapidly spread across Guangdong, China. This outbreak resulted in 8,000 infections and 774 fatalities in 37 countries [9]. The outbreak in $\mathbf{2 0 2 0}$ has presented in the form of pneumonia.

Citation: Jain R, Shukla S, Nema N, Panday A (2020) A Systemic Review: Structural Mechanism of SARSCoV-2A and Promising Preventive Cure by Phytochemicals. Int J Immunol Immunother 7:051. doi. org/10.23937/2378-3672/1410051

Received: May 17, 2020: Accepted: June 22, 2020: Published: June 24, 2020

Copyright: (c) 2020 Jain R, et al. This is an open-access article distributed under the terms of the Creative Commons Attribution License, which permits unrestricted use, distribution, and reproduction in any medium, provided the original author and source are credited. 


\section{Distribution of cases}



Figure 1: Worldwide distribution of COVID-19 cases [5].

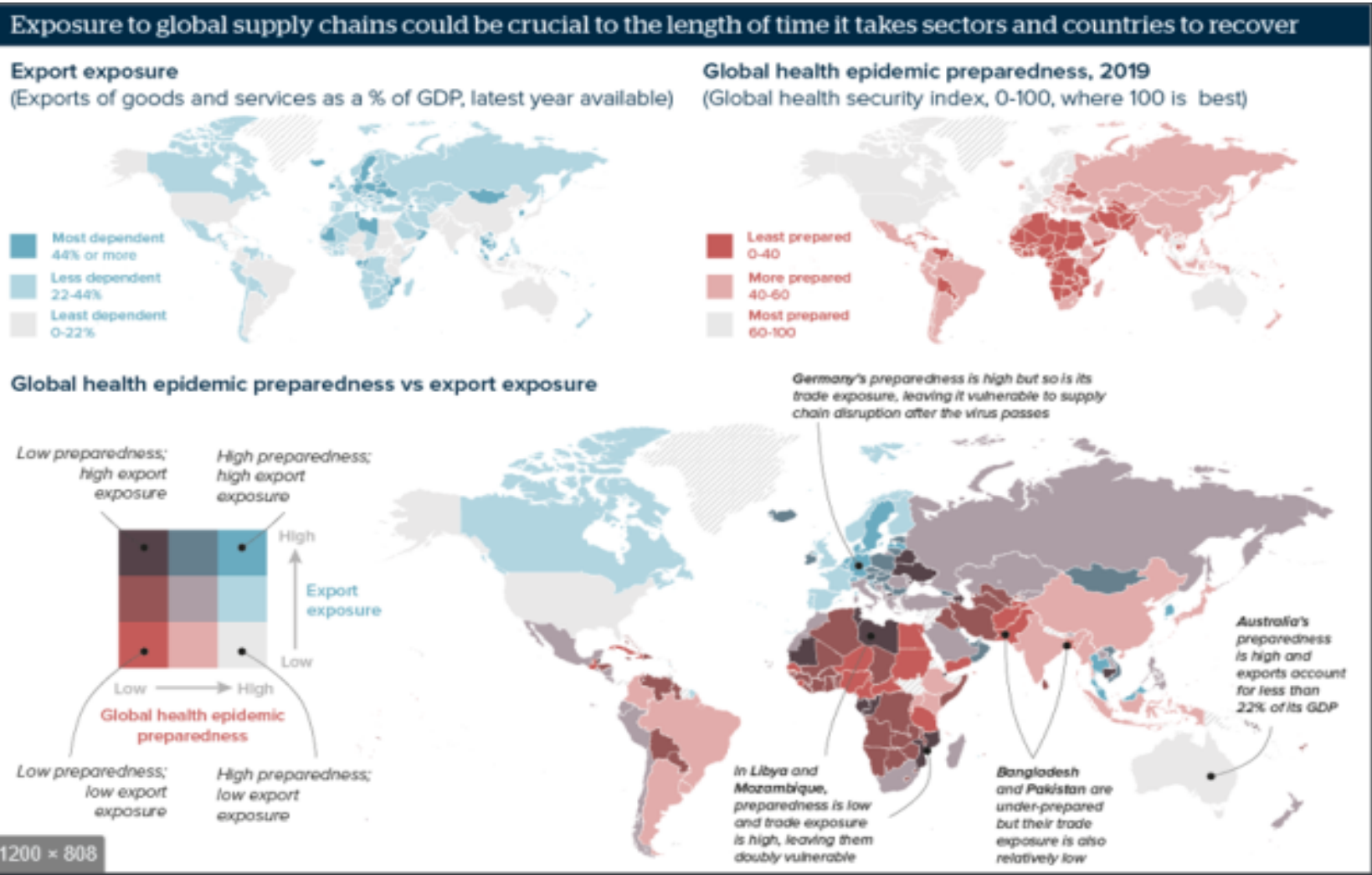

Figure 2: Health preparedness and trade affect COVID-19 exposure [8].

Initially, this virus was designated as 2019-nCoV. However, the International Committee on Taxonomy of Viruses designated it as the SARS-CoV-2 virus [10-13].

The aids epidemic offers an example the broad goal of course is to end the disease the highest leverage approach is prevention. The ideal technology would be vaccine that gives lifelong immunity with a single. When whole world community of scientist working hard to find out clinical solution of this problem so mean time we have to work with what we have in hand the best prevention approach and the phytonutrient could be a one of them.
Phytochemicals are a powerful group of compounds, belonging to secondary metabolites of plants and including a diverse range of chemical entities such as polyphenols, flavonoids, steroidal saponins, organ Sulphur compounds, and vitamins. The potential biological benefits such as, antioxidant, anti-inflammatory, anticancer, antibacterial, antifungal and antiviral activities. The high bioavailability and low cytotoxicity of natural phytochemical it would be great possible candidate in this situation. Here author intended to discuss the mechanism of antiviral properties of few phytochemical toward the SARS-COVID-19 and how it would be a preventive approach in the pandemic situation [14-17]. 




Figure 3: Mechanism of COVID-19 Virus infect to host cell [21].

A



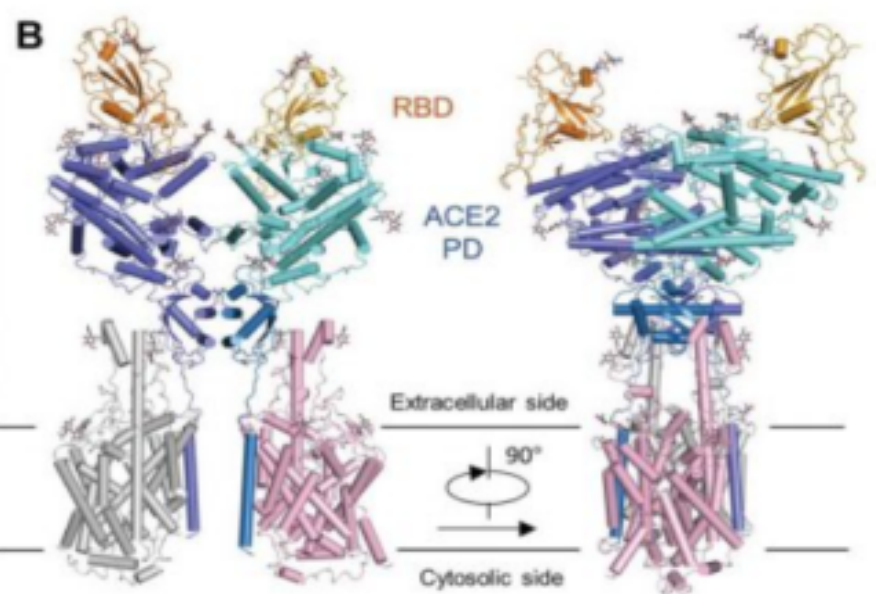

The overall structure of the RBD-ACE2-B0AT1 complex. (A) Cryo-EM map of the RBD-ACE2-BOAT1 complex. Left: Overall reconstruction of the ternary complex at 2.9 A. Inset: focused refined map of RBD. (B) Overall structure of the RBD-ACE2BOAT1 complex. The complex is coloured by subunits, with the protease domain (PD) and the Collectrin-like domain (CLD) coloured cyan and blue in one of the ACE2 protomers, respectively. The glycosylation moieties are shown as sticks (credit: Yan, $\mathrm{R}$ et al..).

Figure 4: Structure of RBD-ACE2 and mechanism how to structurally attached with host cell.

\section{Clinical Mechanism of SARS Spike Protein in Host Cell}

Coronavirus (CoV) is a large family of positive-sense, single-stranded RNA virus that. Their viral RNA genome ranges from 26 to 32 kilobases in length [18]. The virus has four important structural proteins which are $(E)$ the envelope protein $(\mathrm{M})$ the membrane protein $(\mathrm{S})$ the spike protein and $(\mathrm{N})$ the nucleocapsid protein, which are required to regulate the function and viral structure [19] among these virus $S$ and $N$ will help to attachment of virus with host cells. The attachment of virus to the host cells [20].

The S protein has three major sections which are the large ectodomain, a single-pass trans-membrane anchor and a short intracellular tail. These play a major role in anchoring the host cells. Among these sections have two subunits which are the S1 receptor-binding subunit and $\mathrm{S} 2$ the membrane fusion subunit.

During infection, the $S$ protein is cleaved into subunits, S1 and S2. S1 contains the receptor binding domain (RBD) which allows coronaviruses to directly bind to the peptidase domain (PD) of ACE2. S2 then likely plays a role in membrane fusion [20,21] (Figure 3).

Studies, suggests ACE2 needs to dimerism to be active. The resultant homodimer has two PDs, able to bind two COVID-19 S protein trimmers simultaneously in the 
current study, the team identified that the structures could only bind if the PD interacts with the up RBD [22].

The paper, published in Science, suggests ACE2 needs to dimerize to be active. The resultant homodimer has two PDs, able to bind two COVID-19 S protein trimers simultaneously in the current study, the team identified that the structures could only bind if the PD interacts with the up RBD (Figure 4).

They further compared how SARS-CoV-2-RBD binding is different to other SARS-CoV-RBDs binding; showing that some changes in the sequence may make associations tighter in COVID-19, while others could reduce the binding affinity.

This virus belongs to $\beta$-coronavirus, a large class of viruses prevalent in nature. Similar to other viruses, SARS-CoV-2 has many potential natural hosts, intermediate hosts and final hosts.

Cytokine release syndrome is a vital factor that aggravates disease progression. A higher levels of IL- 6 and IL-10, and lower levels of CD4+T and CD8+T are observed in COVID-19 patients parallel with the severity of the disease [7].

\section{Mechanism of Antiviral Activities of Phytoche- micals}

Phytochemical compound works as a legend and protein at the site where it was known to get involved in host cell binding. Their binding interaction is more stable than with that of recently available drug. Similar$l y$, it was also seen that these molecules attach to those sites of ACE2 which were involved in serving a medium of viral entry. Thus, it is apparent from the present study that viral infection can be prevented by Phytochemicals.

Phytochemicals bind to $S$ this would rather serve dual inhibitory machinery by blocking host cell receptor to virus and viral protein entry. Moreover, some of the phytochemicals are more potent immunostimulant and have been reported to induce autophagy, another important mechanism of viral clearance that promotes elimination or neutralisation of viral infection [23].

\section{Blocking mechanism by the Activity of viral RNA}

Some of the myricetin, scutellarein, and phenolic compounds work as natural inhibitors against the SARS-Covid-19. They show the different antiviral mechanisms against SARS-CoV that include inhibiting the viral $3 \mathrm{CL}$ protease and blocking the activity of viral RNA-dependent RNA polymerase29 (Figure 5).

Molecular docking is a computational method which aims to identify non-Covalent binding between protein (receptor) and a small molecule (ligand/Inhibitor). Docking predicts the mode of interaction between a target protein and a small ligand for an established binding site [24].

Phytochemical compound works as a legend. The SARS-CoV-2S is a surface glycoprotein. This protein plays important roles during viral attachment, fusion and entry into the host cells [25]. Biologically this protein exists in a heterotrimeric form with three separate polypeptide chains: Chain A, B and C, forming each monomer [26]. Ligands to investigate their binding affinity with SARS-CoV-2S chain A as the receptor target protein $[21,26,27]$.

\section{Therapeutic Activity of Few Phytochemical-SARS COVID-19}

Medicinal plant compounds are already used to successfully treat numerous viral diseases. Currently no effective treatment is available for COVID-19. Phytochemicals could be an effective tool by increase the Immunity and can transform in to the clinical drug. Thus, it is necessary to further examine the topic of antiviral phytochemicals, highlighting drug delivery applications



Figure 5: Schematic illustration of a viral transmission (Corona virus) replication cycle and infection on the pathogenesis of viral infection. 
in overcoming the multiple biological barriers existing for antiviral agents to successfully reach their intended site(s) of action. Here author focus to explain about few phytochemical they could be a next possible treatment against the pandemic situation.

Ginger, Zingiber officinale, is a widely used has been found active against Human Respiratory Syncytial Virus (HRSV)-induced plaque formation on the epithelium of the airways through blocking viral attachment and internalization. Interestingly, an extract of Pelargonium sidoides reduces rhinovirus infection through modulation of viral binding proteins on human bronchial epithelial cells. For the management of acute bronchitis and acute respiratory tract infections [28].

It's reported these natural compounds effectively prevent the early stage of HCoV-22E9 infection, including viral attachment and penetration. Saikosaponins $(A, B 2, C$, and D), which are naturally occurring triterpene glycosides isolated from medicinal plants such as Bupleurum spp. Heteromorpha spp., and Scrophularia scorodonia exert antiviral activity against HCoV-22E9. Natural inhibitors against the SARS-CoV enzymes, such as the nsP13 helicase and $3 C L$ protease, have been identified as well and include myricetin, scutellarein, and phenolic compounds from Isatis indigotica and Torreya nucifera [29-38] (Table 1).

Table 1: Phytochemical source and mode of action.

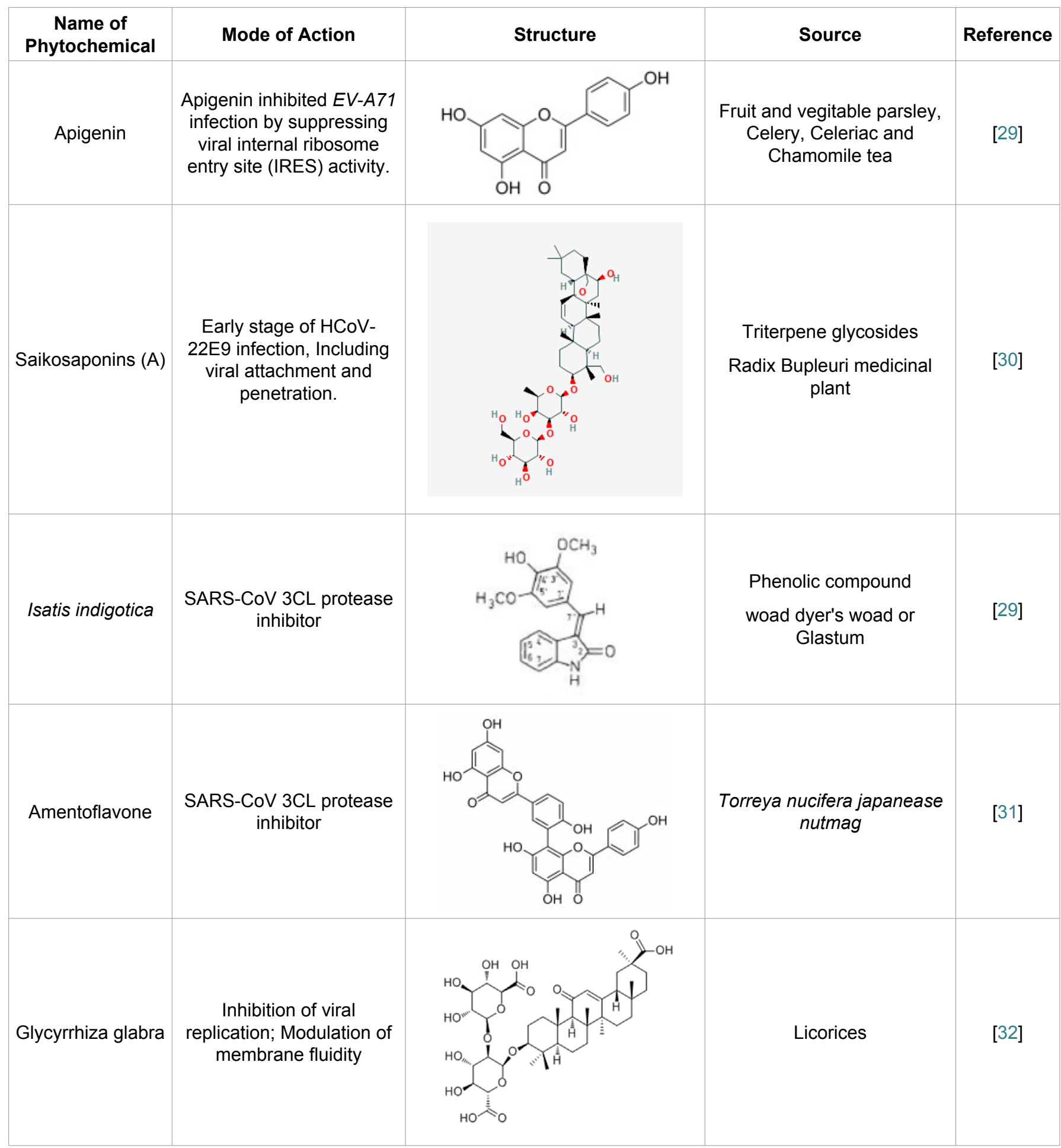




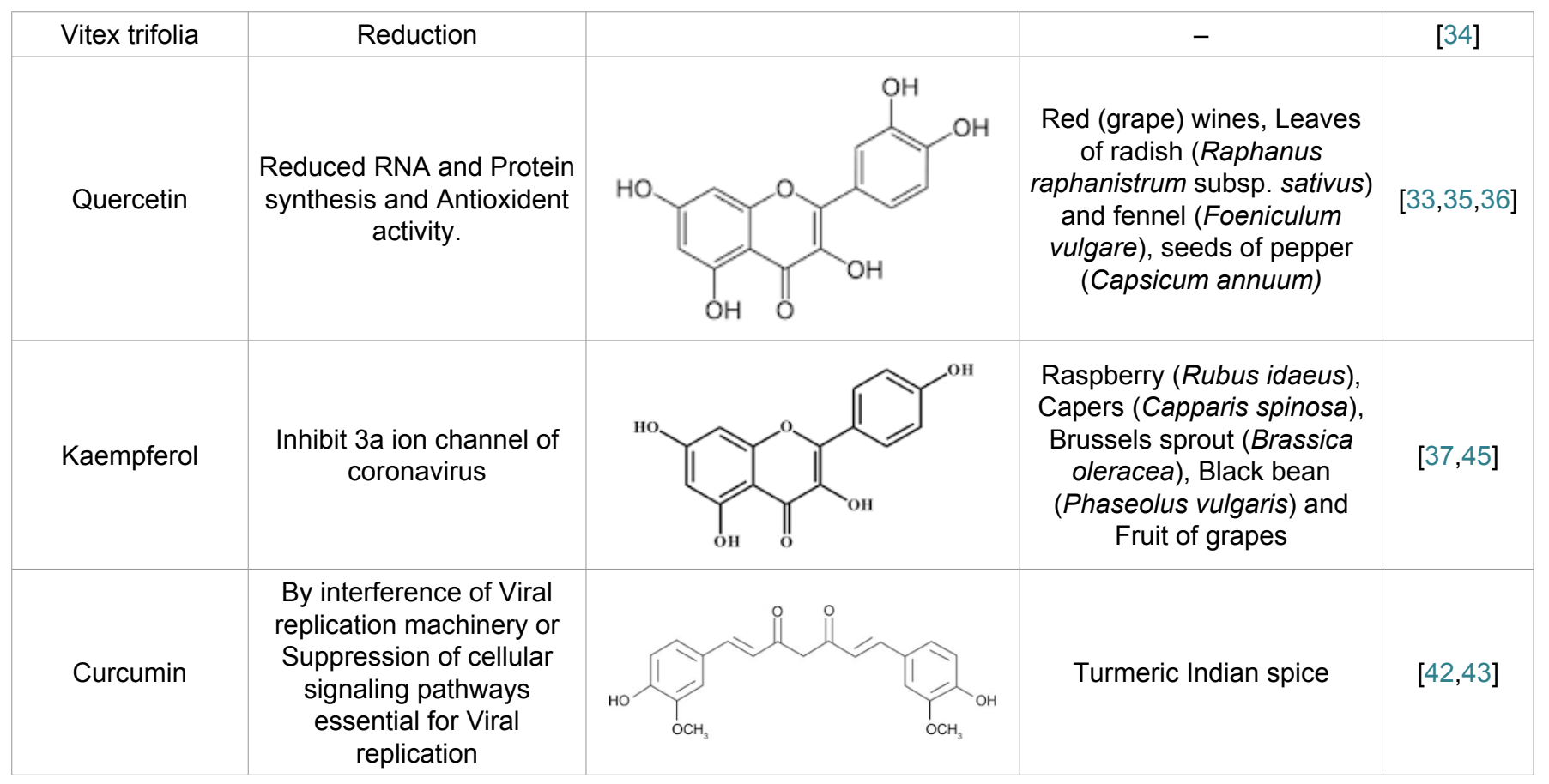

\section{Ashwagandha (W. somnifera)}

It has been reported $W$. somnifera compound, with anone, docked very well in the binding interface of AEC2-RBD complex, and was found to move slightly towards the interface center on simulation. With anone significantly decreased electrostatic component of binding free energies of ACE2-RBD complex. Two salt bridges were also identified at the interface; incorporation of with anone destabilized these salt bridges and decreased their occupancies. We postulate, such an interruption of electrostatic interactions between the RBD and ACE2 would block or weaken COVID-19 entry and its subsequent infectivity [39].

\section{Curcumin}

Due to the lack of preventive and therapeutic options for many viral infections, numerous studies have been conducted to investigate the antiviral potential of various natural compounds $[25,40,41]$. Accumulated evidence indicated curcumin plays an inhibitory role against infection of numerous viruses including hepatitis viruses, influenza viruses and emerging arboviruses like the Zika virus(ZIKV) or chikungunya virus (CHIKV), human immunodeficiency virus (HIV), herpes simplex virus 2 (HSV-2) and human papillomavirus (HPV) [20]. Various studies shows the mechanisms involve either a direct interference of viral replication machinery or suppression of cellular signaling pathways essential for viral replication, such as PI3K/Akt, NF-KB [21]. Inosine monophosphate dehydrogenase (IMPDH) enzyme due to rate-limiting activity in the de novo synthesis of guanine nucleotides is suggested as a therapeutic target for antiviral compounds $[42,43]$.

\section{Glycyrrhizin}

Glycyrrhizin inhibits SARS-associated coronavirus (SARS-CoV) replication in Vero cells with a selectivity in- dex of 67. In addition to inhibition of virus replication, glycyrrhizin is able to inhibit adsorption and penetration of the virus during the early steps of the replicative cycle. The studies from show that glycyrrhizin induces nitrous oxide synthase in Vero cells and that virus replication is inhibited when a nitrous oxide dono r(DETANonoate) is added to the culture medium $[32,35,44]$.

\section{Quercetin}

Quercetin compound with phenolic group occurs widely in nature, found in berries, fruits, grains, onion, kale and vegetables. It neutralizes potentially damaging free radicals through the donation of a hydrogen atom. The antioxident activity is a key interest in current situation. Computer modeling techniques to identify these molecules as conceivably block the "ACE2 receptors" on cells to which the coronavirus that causes COVID-19 attaches, much like a key fits into a lock. Once the virus enters a cell it hijacks the cellular machinery and uses it to reproduce and crank out more viruses. Based on its molecular structure, quercetin could be a "key" that fits the lock and blocks other "keys" [37].

Studies revels kaempferol has high bioavailability. In particular, The fact that these drugs not only block the 3a channel, thus counteracting virus production, but that they also interfere with other steps of the viral life cycle emphasizes the importance of multi-target phytochemical. The glycosides of kaempferol seem to be highly potent candidates for development as anti-coronaviral agents $[37,38,45]$.

\section{Conclusion}

This is an unfortunate truth that a lot of world population will contract SARS-CoV infection. While specific treatment is not yet coming soon, individual preventive and protective measures drive the personal risk of getting the disease. Due to high bioavailability, efficacy 
phytochemicals is a promising preventive option in this situation. Include phytochemical rich food like curcumin, Ginger below the daily intake limit could be prevent the un-effected population of world by the pandemic situation. Author not support to take any kind of those .more attention, research development and clinical trial would be need to became a rising clinical option and development of safe drugs. Authors promote to avoid COVID-19 in addition to a healthy diet, following precautions provided by the Centers for Disease Control and Prevention can help keep you safe.

\section{Conflicts of Interest}

The authors report no conflicts of interest in this work.

\section{Acknowledgements}

I am thankful to our coworkers, Professor Archana Panday and the Department of chemistry Dr. Hari Singh Gour University, India providing an opportunity to work here as a research Scholar.

\section{References}

1. Nimesh Sing, Archna Panday (2020) Corona Virus: A review article to identify novel drug for treatment. IJSR.

2. Jasper Fuk-Woo Chan, Shuofeng Yuan, Kin-Hang Kok, Kelvin Kai-Wang To, Hin Chu, et al. (2020) A familial cluster of pneumonia associated with the 2019 novel coronavirus indicating person-to-person transmission: A study of a family cluster. Lancet 395: 514-523.

3. Chaolin Huang, Yeming Wang, Xingwang Li, Lili Ren, Jianping Zhao, et al. (2020) Clinical features of patients infected with 2019 novel coronavirus in Wuhan, China. Lancet 395: 497-506.

4. (2020) Did pangolins spread the China coronavirus to people?.

5. https://www.nature.com/articles/d41586-020-00364-2

6. https://www.worldometers.info/coronavirus/

7. (2020) Morning star view: The impact of coronavirus on the economy.

8. (2020) Health preparedness and trade affect COVID-19 exposure.

9. (2020) Scientists demonstrate how COVID-19 infects human cells.

10. Li-sheng Wang, Yi-ru Wang, Da-wei Ye, Qing-quan Liu (2020) Review of the 2019 Novel Coronavirus (COVID-19) based on current evidence. International Journal of Antimicrobial Agents.

11. Organization WH (2020) Coronavirus disease (COVID-2019) situation reports

12. Chan JF, To KK, Tse H, Jin DY, Yuen KY (2013) Interspecies transmission and emergence of novel viruses: Lessons from bats and birds. Trends Microbiol 21: 544-555.

13. Zhu N, Zhang D, Wang W, Li X, Yang B, et al. (2020) A novel coronavirus from patients with pneumonia in China, 2019. The New England Journal of Medicine.

14. Thevarajan I, Nguyen THO, Koutsakos M, Julian Druce, Leon Caly, et al. (2020) Breadth of concomitant immune re- sponses prior to patient recovery: A case report of non-severe COVID-19. Nat Med.

15. Forni C, Facchiano F, Bartoli M, Pieretti S, Facchiano A, et al. (2019) Beneficial role of phytochemicals on oxidative stress and age related diseases. BioMed Research International.

16. Panche AN, Diwan AD, Chandra SR (2016) Flavonoids: An overview. Journal of Nutritional Science.

17. Kumar S, Pandey AK (2013) Chemistry and biological activities of flavonoids: An overview. The Scientific World Journal.

18. Hassan S, Sheikh FN, Jamal S, Jude K Ezeh, Ali Akhtar (2020) Coronavirus (COVID-19): A review of clinical features, diagnosis, and treatment. Cureus 12: e7355.

19. D Schoeman, BC Fielding (2019) Coronavirus envelope protein: Current knowledge. Virology J.

20. AC Walls, YJ Park, MA Tortorici, A Wall, AT McGuire, et al. (2020) Structure, function, and antigenicity of the SARSCoV-2 spike glycoprotein. Cell.

21. Balachandar Vellingiri, Kaavya Jayaramayyab, Mahalaxmi lyer, Arul Narayanasamy, Vivekanandhan Govindasamy, et al. (2020) COVID-19: A promising cure for the global panic. Science of the Total Environment.

22. Campbell GR, Pasquier E, Watkins J, Bourgarel-Rey V, Vincent Peyrot, et al. (2004) The glutamine-rich region of the HIV-1 Tat protein is involved in T-cell apoptosis. J Biol Chem 279: 48197-48204.

23. Zhou Y, Simmons G (2012) Development of novel entry inhibitors targeting emerging viruses. Expert review of anti-infective therapy 10: 1129-1138.

24. DeLano W (2002) The PyMOL molecular graphics system. Schrodinger LLC.

25. Steinmann J, Buer J, Pietschmann T, Steinmann E (2013) Anti-infective properties of epigallocatechin-3-gallate (EGCG), a component of green tea. $\mathrm{Br} \mathrm{J}$ Pharmacol 168: 1059-1073.

26. Rane Jitendra Subhash, Chatterjee Aroni, Kumar Abhijeet, Ray Shashikant (2020) Targeting SARS-CoV-2 Spike Protein of COVID-19 with naturally occurring phytochemicals: An in silco study for drug development. ChemRxiv.

27. Belouzard S, Millet JK, Licitra BN, Whittaker GR (2012) Mechanisms of coronavirus cell entry mediated by the viral spike protein. Viruses 4: 1011-1033.

28. https://www.researchsquare.com/article/rs-22057/v1

29. Thomas T Yoshikawa, Kevin P (2001) Nutritional strategies to boost immunity and prevent infection in elderly individuals. High Clinical Infectious Diseases 33: 1892-1900.

30. Zorofchian Moghadamtousi S, Hajrezaei M, Abdul Kadir H, Zandi K (2013) Loranthus micranthus Linn: Biological activities and phytochemistry. Evidence-Based Complementary and Alternative Medicine.

31. (2013) World Health Organization.

32. SY Li, C Chen, HQ Zhang, HY Guo, H Wang, et al. (2005) Identification of natural compounds with antiviral activities against SARS-associated coronavirus. Antivir Res 67: 1823.

33. C Fiore, M Eisenhut, R Krausse, E Ragazzi, D Pellati, et al. (2008) Antiviral effects of Glycyrrhiza species. Phytother Res 22: 141-148. 
34. J Liou, CY Cheng, KW Yeh, YH Wu, WC Huang (2018) Protective effects of casticin from vitextrifoliaalleviatee osinophilic airway inflammation and oxidative stress in a murine asthma model. Front Pharmacol 635.

35. Salima Lalani, Chit Laa Poh (2020) Flavonoids as Antiviral Agents for Enterovirus A71 (EV-A71). Viruses 12: 184.

36. Cinatl J, Morgenstern B, Bauer G, Chandra P, Rabenau H, et al. (2003) Glycyrrhizin, an active component of liquorice roots, and replication of SARS-associated coronavirus. The Lancet 14: 2045-2046.

37. Liang-TzungLin, Wen-ChanHsu, Chun-ChingLin (2014) Antiviral natural products and herbal medicines. J Tradit Complement Med.

38. https://www.mcgill.ca/oss/article/health/quercetin-take-or-not-take

39. Chandel V, Raj S, Rathi B, Kumar D (2020) In Silico Identification of Potent COVID-19 main protease inhibitors from FDA approved antiviral compounds and active phytochemicals through molecular docking: A drug repurposing approach. Preprints.
40. (2020) European Review for Medical and Pharmacological Sciences 24

41. Acharya Balkrishna, Subarna Pokhrel, Jagdeep Singh, Anurag Varshney (2020) Withanone from Withania somnifera May Inhibit Novel Coronavirus (COVID-19) Entry by Disrupting Interactions between Viral S-Protein Receptor Binding Domain and Host ACE2 Receptor.

42. Dimas Praditya, Lisa Kirchhoff, Janina Bruning, Heni Rachmawati, Joerg Steinmann (2019) Anti-infective Properties of the Golden Spice Curcumin. Front Microbiol.

43. Mathew D, Hsu WL (2018) Antiviral potential of curcumin. Journal of Functional Foods.

44. CW Lin, FJ Tsai, CH Tsai, CC Lai, L Wan, et al. (2005) Anti-SARS coronavirus $3 \mathrm{C}$-like protease effects of Isatisindigotica root and plant-derived phenolic compounds. Antivir Res 68: 36-42.

45. Silvia Schwarz, Daniel Sauter, Kai Wang, Ronghua Zhang, Bing Sun, et al. (2014) Kaempferol Derivatives as Antiviral Drugs against the $3 a$ Channel Protein of Coronavirus. Planta Med. 